\title{
Auf der letzten Meile zum Patienten
}

\author{
Aref AI-Deb'ia, Annette Eggera, Peter Indra ${ }^{a}$, Christoph Rödert ${ }^{b}$ \\ ${ }^{\text {a } G e s u n d h e i t s d e p a r t e m e n t ~ d e s ~ K a n t o n s ~ B a s e l-S t a d t, ~ B e r e i c h ~ G e s u n d h e i t s v e r s o r g u n g ; ~}{ }^{b}$ Universität Bern, Institut für Evaluative Forschung in der Medizin (IEFM)
}

Die drei Dimensionen von Struktur-, Prozess-, und Ergebnisqualität gehören zu unserem Alltag in der Qualitätssicherung der stationären Gesundheitsversorgung. In einem nächsten Schritt wird nun das Versorgungsmonitoring auf kantonaler Ebene gestärkt und um die vierte Qualitätsdimension, die Indikationsqualität von Hüft- und Knie-Totalendoprothesen (TEP), erweitert.

\section{Hintergrund}

Im spitalübergreifenden Qualitätsmonitoring des Kantons Basel-Stadt (QuBA) [1] werden seit dem Jahr 2009 erfolgreich Themen für die Qualitätssicherung aller Spitäler und Kliniken des Kantons (Akutsomatik, Psychiatrie und Rehabilitation) ausgewählt, Massnahmen umgesetzt und die Ergebnisse publiziert [2, 3]. Die aktive Teilnahme am Qualitätsmonitoring ist verpflichtender Bestandteil des Leistungsauftrages, den das Gesundheitsdepartement im Kanton Basel-Stadt derzeit an drei Universitätsspitäler und zehn Grundversorgungs- und Spezialkliniken erteilt. In einem nächsten Schritt wird nun das Versorungsmonitoring auf kantonaler Ebene gestärkt und neben der Struktur-, Prozess-, und Ergebnisqualität um eine vierte Dimension, die Indikationsqualität, erweitert.

\section{Problemstellung und Ausgangslage}

Die Indikationsstellung bei Erstimplantationen und Revisionen von Hüft- und Knie-Totalendoprothesen und deren Mengenentwicklung wird in der Schweiz,

Die Schweiz nimmt bei der Häufigkeit des Hüftgelenkersatzes im Vergleich der OECDLänder den ersten Platz ein.

wie auch international, kritisch diskutiert. Beim direkten Vergleich mit anderen Ländern zur Eingriffshäufigkeit findet sich die Schweiz im internationalen Spitzenfeld wieder [4, 5]. Gemäss den Auswertungen der OECD (Organisation for Economic Cooperation and Development) wurden im Jahr 2011 in der Schweiz 306 Hüft-Endoprothesenimplantationen pro 100000 Einwohner durchgeführt (Abb. 1). Damit nimmt die Schweiz bei der Häufigkeit des Hüftgelenkersatzes im Vergleich der OECD-Länder den ersten Platz ein. Im Hinblick auf die Knie-Endoprothesenimplantationen landet die Schweiz gemäss der OECD-Auswertung für das Jahr 2011 mit 205 Eingriffen pro 100000 Einwohner hinter

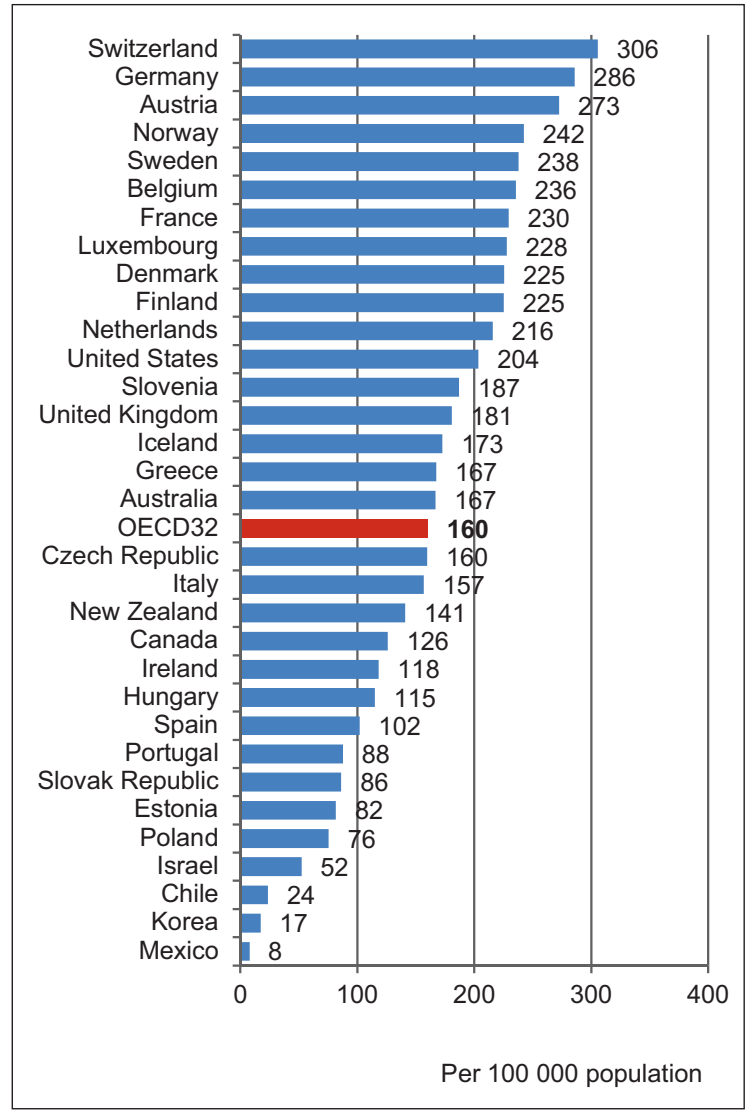

Abbildung 1: Häufigkeit des Hüftgelenkersatzes im OECDVergleich für das Jahr 2011 (bzw. für das aktuellste zur Verfügung stehende Datenjahr der jeweiligen Länder) [4]. 


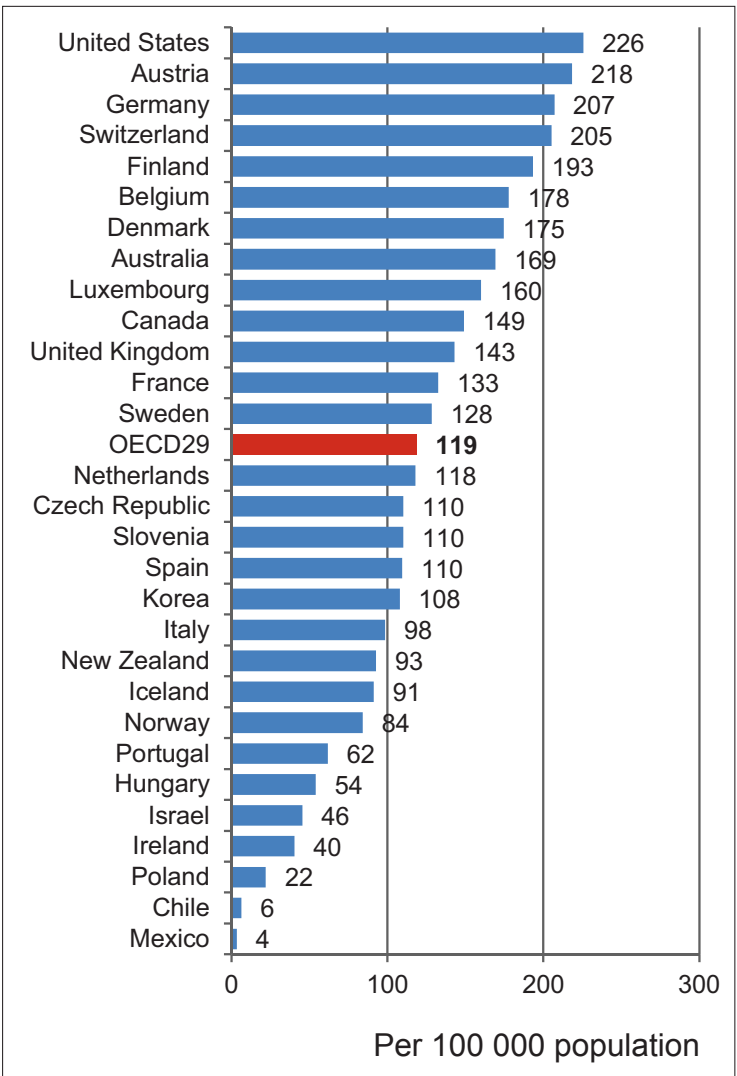

Abbildung 2: Häufigkeit des Kniegelenkersatzes im OECDVergleich für das Jahr 2011 (bzw. für das aktuellste zur Verfügung stehende Datenjahr der jeweiligen Länder) [4].

den USA, Österreich und Deutschland auf dem vierten Platz im Häufigkeitsranking (Abb. 2).

Gemäss den Auswertungen der OECD weist die Schweiz auch für die Vorjahre relativ hohe Eingriffshäufigkeiten beim Hüft- und Kniegelenkersatz auf, die im Zeitraum von 2000 bis 2011 kontinuierlich zugenommen haben (Abb. 3 und 4).

Allerdings ist die Interpretierbarkeit dieser internationalen OECD-Vergleiche eingeschränkt, da die unterschiedlichen demographischen Bevölkerungsstrukturen, die Unterschiede zwischen den einzelnen Gesundheitssystemen und der damit verbundene Zugang zu Gelenkersatzeingriffen unberücksichtigt bleiben und die Kodierung bzw. Berechnung der Eingriffszahlen in den verschiedenen Ländern nicht einheitlich erfolgt [4-6]. Ob in der Schweiz bzw. im Kanton Basel-Stadt eine angemessene Anzahl (appropriateness) an Hüft- oder Knie-Totalendoprothesen eingesetzt wird und inwieweit eine Über-, Unter- oder Fehlversorgung vorliegt, muss mit Langzeitstudien untersucht werden. Idealerweise wird eine solche Studie an ein Register gekoppelt und der patientenbezogene medizinische Nutzen mit Zielgrössen wie funktionalem Ergebnis oder Lebensqualität gemessen.

\section{Projektbeschreibung}

Im Schweizerischen Implantat-Register (SIRIS) [7] werden seit dem Jahr 2012 die Implantationen und Revisionen von Hüft- und Knie-Totalendoprothesen erfasst, zusätzlich kann innerhalb von SIRIS der entsprechende patientenbezogene Nutzen auf freiwilliger Basis miterhoben werden. Im Rahmen unseres Projekts soll nun zusätzlich der prä- und postoperative krankheitsspezifische Zustand von Patientinnen und Patienten, die eine Erstimplantation oder Revision einer Hüft- oder Knieprothese in einem basel-städtischen Spital erhalten, gemessen werden. Hierzu wird das SIRIS-Register als inhaltliche und technische Basis genutzt. Da das SIRIS als Prothesenregister eher den technischen Erfolg der Operation misst, nämlich die Überlebenszeit der Prothese und weitere Revisionseingriffe, soll in einer zunächst dreijährigen Messphase auch die Sicht des Patienten erfasst werden, um damit annäherungsweise die Indikations- und Ergebnisqualität bestimmen zu können. Eine wissenschaftlich validierte und einheitliche Messung des medizinischen Nutzens von Erstimplantationen und Revisionen von Hüft- und Knie-Totalendoprothesen ist anhand des Core Outcome Measures Index-(COMI-)Patientenfragebogens möglich [8]. Dieser misst zu den Bereichen Schmerzintensität, Gelenkfunktion, symptomspezifisches Wohlbefinden, Lebensqualität und Einschränkung bei der Ausübung der gewohnten Tätigkeiten im Alltag (Arbeit, Hausarbeit, Schule, Freizeitaktivitäten) vor und nach einem

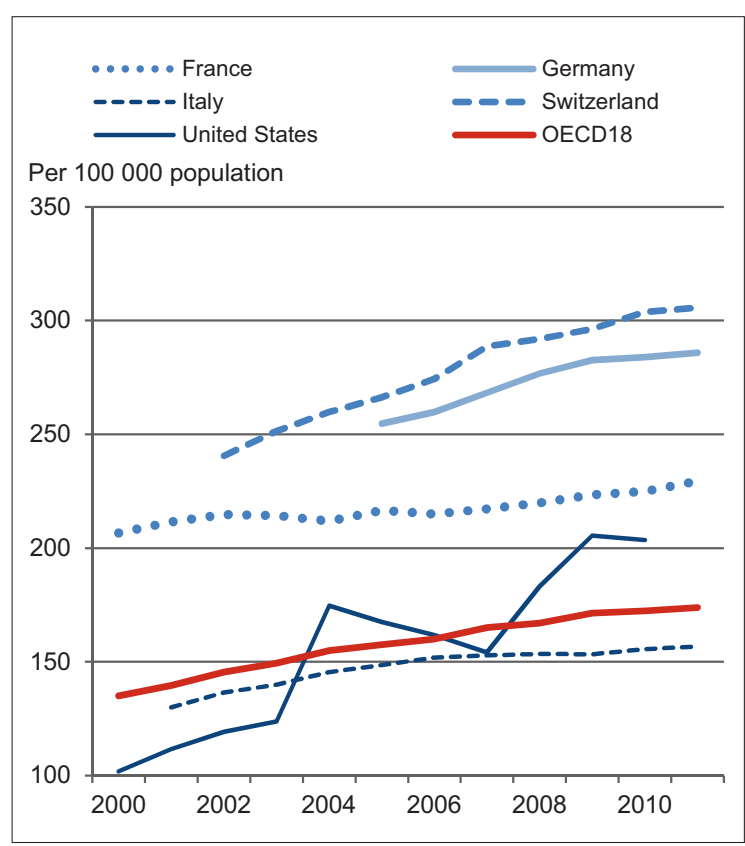

Abbildung 3: Entwicklung der Eingriffshäufigkeit beim Hüftgelenkersatz in ausgewählten OECD-Ländern für die Jahre 2000 bis 2011 (bzw. für das aktuellste zur Verfügung stehende Datenjahr der jeweiligen Länder) [4]. 


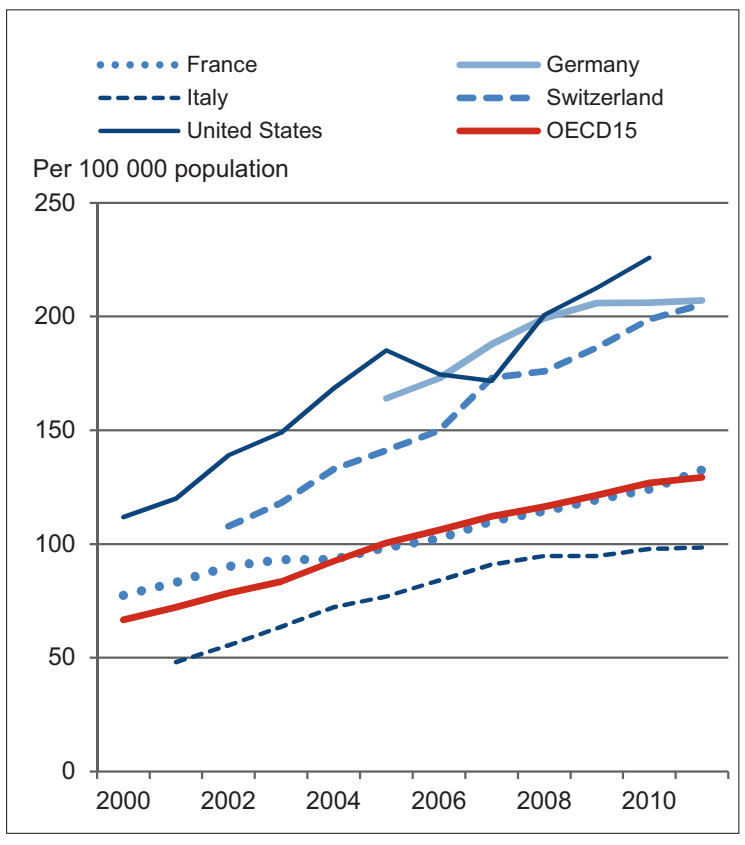

Abbildung 4: Entwicklung der Eingriffshäufigkeit beim Kniegelenkersatz in ausgewählten OECD-Ländern für die Jahre 2000 bis 2011 (bzw. für das aktuellste zur Verfügung stehende Datenjahr der jeweiligen Länder) [4].

orthopädischen Eingriff am Hüft- oder Kniegelenk die Wirksamkeit und Indikationsqualität der Behandlung. Darüber hinaus werden die behandelten Patientinnen und Patienten in den Fragebögen nach der Operation zusätzlich nach aufgetretenen Komplikationen (wie z.B. Störung der Wundheilung, Lähmung, Gefühlsstörungen, Muskelverhärtung), erneut durchgeführten Operationen, der Behandlungszufriedenheit und dem subjektiv empfundenen Nutzen des operativen Eingriffs befragt, womit neben der Indikations- auch die Behandlungsqualität erfasst wird [8, 9]. Die Indikationsstellung für die Operation und vor allem ihr Zeitpunkt sind neben der Prozessqualität des eigentlichen Eingriffs wichtige Prädiktoren für das Behandlungsresultat. Ein wesentlicher Vorteil des COMI-Patientenfragebogens ist die Kombination aus prä- und postoperativer Befragung. Die Antworten werden unabhängig von der Operateurin oder dem Operateur von einem neutralen Institut erhoben und ausgewertet. Mögliche Einflüsse der Arzt-Patienten-Beziehung auf die Diagnose- und Indikationsqualität werden durch das Ausfüllen des Fragebogens nach dem Spitalaufenthalt im heimischen Umfeld umgangen. Durch aktivere Teilnahme am Prozess der Indikationsstellung wird der/ die betroffene Patient/Patientin in seiner/ihrer Gesundheitskompetenz sensibilisiert und gestärkt.

In Zusammenarbeit mit dem Institut für Evaluative Forschung in der Medizin (IEFM) der Universität Bern werden ab 2017 bei Hüft- und Knie-TEP-Patienten mit- hilfe des validierten COMI-Patientenfragebogens die Schmerzintensität, Funktion, Zufriedenheit und Lebensqualität der betreffenden Patienten präoperativ und 6 bzw. 24 Monate nach der Operation erhoben, um somit letztendlich den medizinischen Nutzen sowie annäherungsweise die Indikations- und Ergebnisqualität der Versorgung bestimmen zu können.

\section{Fazit}

Der Kanton Basel-Stadt möchte mit diesem Projekt seiner Vorreiterrolle beim Qualitäts- und Versorgungsmonitoring in der Schweiz gerecht werden und als erster Kanton einen Beitrag zur Versorgungsforschung in der Schweiz leisten. Die erhobenen Resultate werden einen festen Bestandteil des Qualitätsmonitorings im Kanton Basel-Stadt bilden. Im Weiteren wird auch noch geprüft werden, wo Synergien mit bereits laufenden Qualitätsmessungen von swiss orthopaedics (Schweizerische Gesellschaft für Orthopädie und Traumatologie) und der FMH in diesem Bereich bestehen und allenfalls miteinbezogen werden könnten. Schweizweit sind zudem weitere Kantone daran interessiert, sich an dieser Qualitätsmessung zu beteiligen.

\section{Literatur}

1 Gesundheitsdepartement des Kantons Basel-Stadt, Bereich Gesundheitsversorgung. Qualitätsmonitoring Basel-Stadt. www.gesundheitsversorgung.bs.ch/qualitaet-aufsicht/spital. html [letzter Zugriff: 19.10.2015].

2 Schneider P, Egger A, Kurz R. Bessere Qualität dank Critical Incident Reporting-Systemen? Eine Frage der Kultur? Schweiz Ärztezeitung. 2013;94(37):1407-10.

3 Gesundheitsdepartement des Kantons Basel-Stadt, Bereich Gesundheitsversorgung. Gesundheitsversorgungsbericht des Jahres 2014

www.bs.ch/publikationen/gesundheitsversorgung/gsv-bericht. html [letzter Zugriff: 11.11.2015].

4 OECD (Organisation for Economic Cooperation and Development). Health at a Glance 2013: OECD Indicators, OECD Publishing. 2013. http://dx.doi.org/10.1787/health_glance-2013-en [letzter Zugriff: 19.10.2015].

5 McPherson K, Gon G, Scott M. International Variations in a Selected Number of Surgical Procedures, OECD Health Working Papers, No. 61, OECD Publishing. 2013. http://dx.doi. org/10.1787/5k49h4p5g9mw-en [letzter Zugriff: 19.10.2015].

6 Wengler A, Nimptsch U, Mansky T. Hip and knee replacement in Germany and the USA - analysis of individual inpatient data from German and US hospitals for the years 2005 to 2011. Deutsches Ärzteblatt International. 2014;111:407-16.

7 Stiftung für Qualitätssicherung in der Implantationsmedizin. Schweiz. Implantat-Register (SIRIS). www.siris-implant.ch/de/ [letzter Zugriff: 19.10.2015].

8 Impellizzeri FM, Mannion AF, NaalFD, Leunig M. A Core Outcome Measures Index (COMI) for patients undergoing hip arthroplasty. The Journal of Arthroplasty. 2013;28(9):1681-6.

9 The Spine Society of Europe (EuroSpine). www.eurospine.org/p31000375.html [letzter Zugriff: 19.10.2015].

\section{Bildnachweis}

OECD. Health at a Glance 2013: OECD Indicators, OECD Publishing. 2013. http://dx.doi.org/10.1787/health_glance-2013-en 